\title{
In-situ neutron diffraction study of micromechanical shear failure in an aerospace composite
}

\author{
Bing Wang ${ }^{1}$, Keith A Seffen ${ }^{2}$, Simon D Guest ${ }^{3}$ \\ Department of Engineering, University of Cambridge, Cambridge, CB2 1PZ, UK \\ Tung-Lik Lee ${ }^{4}$ \\ ISIS Neutron and Muon Source, Rutherford Appleton Laboratory, Harwell Oxford, Didcot, OX11 OQX, UK \\ Shi Huang ${ }^{5}$, Shifeng $\mathrm{Luo}^{6}$, Jiawei $\mathrm{Mi}^{7}$ \\ Department of Engineering, University of Hull, Hull, HU6 7RX, UK
}

\begin{abstract}
Glass fibre reinforced polypropylene (GF/PP) composite is a well-established material system for fabricating bistable composite tape-spring (CTS) structure. It is light-weight and multifunctional, and has attracted growing interest in shape-adaptive and energy harvesting systems in defence, civil and especially aerospace engineering. The factors governing its bistability have been well-understood; whist there is limited research concerning the micromechanics and microstructural failure. In this research, we investigate the failure mechanisms of the GF/PP composite. This is achieved by performing in-situ neutron diffraction on composite specimens using the ENGIN-X neutron diffractometer at Rutherford Appleton Laboratory. Shear failures are characterised at both macroscopic and microscopic scales. Elastic and viscoelastic strain evolutions at different levels reveal the micromechanical shear failure. The failure mechanisms are then proposed, which will benefit optimisation of structural design and structural integrity of the CTS in aerospace applications.
\end{abstract}

\section{Nomenclature}

$d_{h k l}=$ Lattice spacing in the $h k l$ crystalline plane

$h \quad=$ Planck's constant

$k_{\beta} \quad=$ Fraction of $\beta$-crystals

$m=$ Neutron mass

$t \quad=$ Time of flight

$A=$ Integrated area of crystalline peak

$G \quad=$ Shear modulus

$H \quad=$ Height of a crystalline peak

I = Intensity of neutron diffraction

$L \quad=$ Flight path of neutrons

$S_{i j} \quad=$ Elements of the compliance matrix

$\varepsilon=$ Strain

$\lambda=$ Wavelength

$\gamma \quad=$ Shear strain

$\theta_{\mathrm{b}} \quad=$ Fixed angle of neutron scanning

\footnotetext{
${ }^{1}$ Research Associate, Department of Engineering, University of Cambridge, AIAA Member 905310.

${ }^{2}$ Reader in Structural Mechanics, Department of Engineering, University of Cambridge, AIAA Member 241666.

${ }^{3}$ Professor in Structural Mechanics, Department of Engineering, University of Cambridge.

${ }^{4}$ Instrument Scientist, ISIS Neutron and Muon Source, Rutherford Appleton Laboratory.

${ }^{5} \mathrm{PhD}$ student, Department of Engineering, University of Hull.

${ }^{6} \mathrm{PhD}$ student, Department of Engineering, University of Hull.

${ }^{7}$ Professor in Materials, Department of Engineering, University of Hull.
} 


\section{Introduction}

Glass fibre reinforced polypropylene (GF/PP) composite is a well-established material system for fabricating bistable composite tape-spring (CTS) structures, which have been extensively used in defence, civil and aerospace applications. The CTS was invented by Daton-Lovett in 1996 [1], and consolidated theoretically by Cambridge researchers [2-5]. The factors governing its bistability are well-understood: it is a combination of material constitutive behaviour, initial geometrical proportions, and geometrically non-linear response [5]. This inherent structural behaviour has similarities with the lock-stay or side-stay assemblies within an aircraft landing gear: these extend and retract with the gear but remain in a fixed position when the gear is stowed in the bay or locked in the down position. Thus, it is envisaged that by using CTS structures, they could further reduce weight, complexity and maintenance compared to the conventional lock-link assemblies [6].

We have characterised the folding nature of the GF/PP-based CTS structures through experiments, finite element analysis and theoretical modelling [7]: a typical folding process consists of linear bending, torsional buckling, localisation and then folding at large displacements [8]; the folded tape shape is dominated by axial strains and transverse curvature changes [9]. To date, there has been extensive research on microstructural analysis of a semicrystalline polymeric material; whist the micromechanical evolution within a composite is rarely studied. Investigations into the latter would provide essential insight for maintaining the structural integrity of the CTS during the folding process. This can be achieved through diffraction techniques.

Non-destructive testing using X-rays or neutron diffraction offer special advantages over conventional destructive methods, and have been extensively used to investigate in-situ, the microstructures, residual stresses, strain and stress fields, deep inside a material system [10]. The strain measurements are based on monitoring the shifts of the Bragg's peak positions within a structure [11]. Although X-rays and neutron diffractions share similar principles, the neutron technique is superior in terms of penetration depth and light elements (with bigger neutron absorption cross-section) detection [12]. The ENGIN-X at Rutherford Appleton Laboratory (STFC, UK) is a dedicated time-of-flight (ToF) neutron diffractometer for strain measurements. It offers a large detector coverage area and wide scattering range, in order to provide data with high stability and accuracy [13]. Thus, it is an ideal tool to study the strain-dependent shear failure of the GF/PP composite.

In this research, we investigate the shear failures of the GF/PP composite at both macroscopic and microscopic scales. The macroscopic failure is characterised through tensile failure of $\pm 45^{\circ}$ laminates; whilst microscopic failure is studied in-situ, by applying neutron beam at different strain levels using ENGIN-X. Here, we highlight the elastic and viscoelastic strain evolutions at both macroscopic and microscopic levels, in order to characterise the fundamentals of micromechanical failure. Failure mechanisms are then proposed to provide further insight to benefit optimisation of structural design, as well as structural integrity and lifetime predictions of the CTS for aerospace applications.

\section{Technical background}

\section{A. Neutron strain scanning}

Following the discovery of the neutron by Sir James Chadwick in the Cavendish Laboratory at Cambridge University in 1932 [14], the neutron strain scanning (NSS) method was developed in the 1960s [10]. Figure 1 schematically shows an experimental setup for the neutron ToF scattering using ENGIN-X. The pulsed neutron beam with a wide range of kinetic energy impinges on a sample and is then scattered by its internal structures (phases); the detectors collect the diffracted neutrons at a fixed angle of $2 \theta_{\mathrm{b}}$. Neutrons can penetrate deeply into a material, and therefore elastic strains can be measured non-destructively as briefly described blow. The wavelength of the detected neutrons is defined by its ToF time, $t[13]$ :

$$
\lambda=\frac{h}{m\left(L_{1}+L_{2}\right)} t
$$

where $h$ is the Planck's constant, $m$ is the neutron mass, $L_{1}$ and $L_{2}$ are the primary and secondary flight paths, respectively, see Figure 1. 


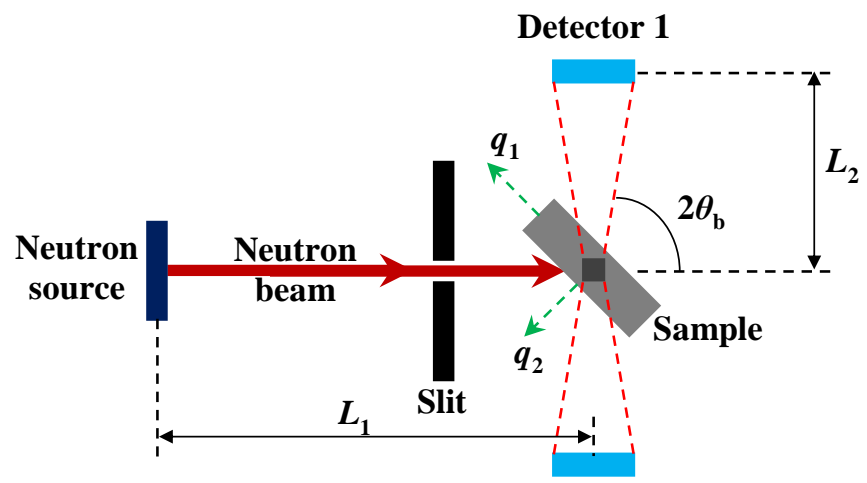

Detector 2

Figure 1 A schematic representation of the principle of neutron strain scanning using ENGIN-X diffractometer; elastic strain is measured along the directions of the impulse exchange vectors, $q_{1}$ (longitudinal direction) and $q_{2}$ (thickness direction), through the two detectors.

The spectrum diffracted by a polycrystalline material consists of different crystalline peaks corresponding to a Miller index $(h k l)$ family of lattice planes, and follow Bragg's law. Thus, the $d$-spacing is obtained from the position $t_{h k l}$ of the peak in the ToF spectrum [13]:

$$
d_{h k l}=\frac{h}{2 \sin \theta_{\mathrm{B}} m\left(L_{1}+L_{2}\right)} t_{h k l}
$$

The peak positions can be determined precisely by a least-square refinement of the peaks, with a typical resolution of $50 \mu \varepsilon$. The elastic strain, $\varepsilon_{h k l}$, is calculated from the changes in the molecular interplanar distances $d_{h k l}$ along a general direction, compared with a stress-free reference $d_{h k l}^{0}$ measured in the same direction:

$$
\varepsilon_{h k l}=\left(d_{h k l}-d_{h k l}^{0}\right) / d_{h k l}^{0}
$$

\section{B. Phase identification}

To determine the phases of the GF/PP composite from neutron scattering, we first scanned pure GF and pure PP samples separately, which contain the same volume of material as in a GF/PP specimen. It shows that diffraction from GF has negligible effect on diffraction pattern of PP: the peak intensity count of pure GF pattern is only 9000 , compared to 230000 in a pure PP sample. Furthermore, the neutron pattern of pure PP is also found effectively the same as in a GF/PP sample.

The PP matrix is a semi-crystalline polymer with a melting temperature $\left(T_{\mathrm{m}}\right)$ of $453 \mathrm{~K}$ and a glass transition temperature $\left(T_{\mathrm{g}}\right)$ of $263 \mathrm{~K}$. Its microstructure has been extensively studied and well-understood [15-17]. The crystalline phase of PP varies from $\alpha$ - to $\gamma$-crystals, depending on the thermal conditions [18]. Generally, cooling of the PP at 50-300 K/s leads to formation of $\alpha$-crystals; at lower than $50 \mathrm{~K} / \mathrm{s}, \beta$-crystals grow. Faster cooling is associated with mesomorphic phase or vitrification of the entire melt [19]. The $\gamma$-crystals develop preferentially in the presence of chain defects, which limit the isotactic sequences, in low molar mass fractions or during crystallisation at an elevated pressure [20].

Figure 2 shows an example of profile analysis of the neutron scattering pattern of a GF/PP sample obtained at ENGIN-X. The 2D neutron scattering data is smoothed first using MDI Jade 6.0 (Materials Data Inc., Livermore, CA, USA), and the background is maintained as linear. The profile function based on Pearson's VII distribution is used for curve-fitting, and expressed as [21]:

$$
I_{2 \theta}=\frac{2 \Gamma(m)\left(2^{1 / m}-1\right)^{1 / 2}}{\sqrt{\pi} \Gamma(m-1 / 2)} \frac{I_{k}}{H_{k}}\left[1+4\left(\frac{2 \theta-2 \theta_{k}}{H_{k}}\right)^{2}\left(2^{1 / m}-1\right)\right]^{-m}
$$

here $m$ is the shape parameter and equal to 1.52, which has been proven to give the best fit for PP [22]. The integrated areas of the crystalline peaks can be used to calculate the crystallinity: 


$$
\text { Crystallinity }=\frac{\sum A_{\text {cri }}}{\sum A_{\text {cri }}+A_{\text {amo }}}
$$

where $\sum A_{\text {cri }}$ is the sum of integrated area of all the crystalline peaks, and $A_{\text {amo }}$ is the area of amorphous halo [18]. Define the relative fraction of $\beta$-crystals, $k_{\beta}$, as:

$$
k_{\beta}=\frac{H_{\beta_{1}}}{H_{\beta_{1}}+H_{\alpha_{1}}+H_{\alpha_{2}}+H_{\alpha_{3}}}
$$

$H_{\alpha_{1}}, H_{\alpha_{2}}, H_{\alpha_{3}}$ are heights of the three strong equatorial $\alpha$-crystalline peaks (110), (040) and (130), respectively; $H_{\beta_{1}}$ is the height of the strong $\beta$-crystalline peak (300). Here, the representative $\beta$-peak is compared to the sum of the three $\alpha$-crystalline peaks rather than any individual of these peaks, since variation in the relative heights of three $\alpha$-peaks depends on the degree of isotaxy and the thermal treatment applied [18].

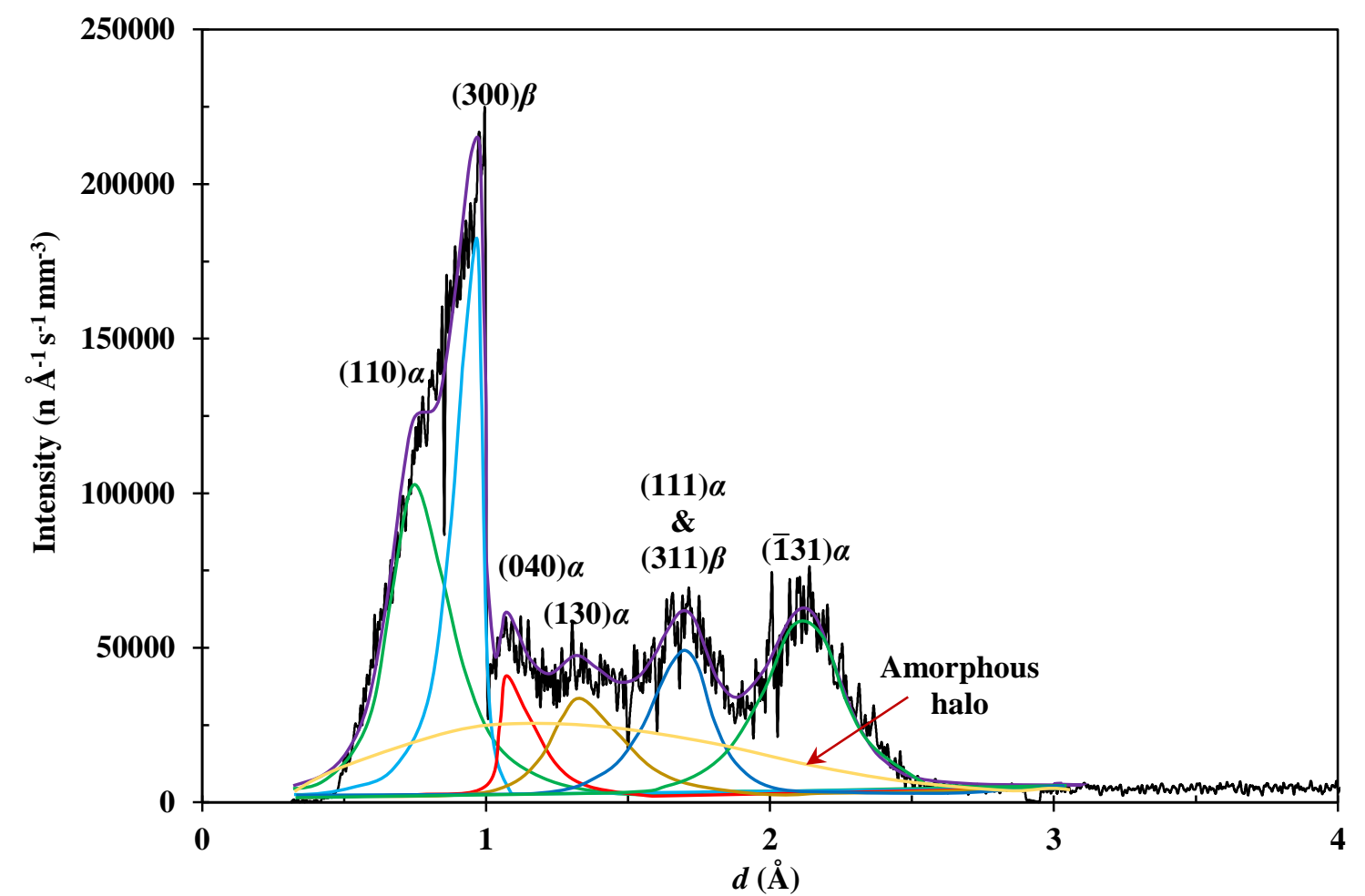

Figure 2 Profile analysis of neutron scattering pattern of a GF/PP composite sample in a stress free state using ENGIN-X.

The phases within a GF/PP composite can be determined as indicated in Figure 2. Upon cooling, the PP matrix crystallises into $\alpha$-crystals. Since the surfaces of oriented $\alpha$-crystals can trigger the formation of $\beta$-crystals [23], the oven-baking process results in a mixture of $\alpha$ - and $\beta$-crystals; the peak positions are also identified [18]. The peak reflections from $\alpha$-crystals include (110), (040), (130), (111) and (131); $\beta$-reflection peaks include (300), and (311). The latter coincides with the $\alpha$-(111) reflection and is invisible; the (300) is distinctive and a strong reflection that usually represents $\beta$-crystals [24]. The crystallinity and fraction of $\beta$-crystals within a non-stressed GF/PP composite can be determined using Eqs. 5 and 6, and they are found to be $70.9 \%$ and $51.0 \%$, respectively. 


\section{Experimental}

\section{A. Composite sample preparation}

Our composite samples use glass fibre (GF) reinforced polypropylene (PP). The layup consists of three layers of plain-weave GF and PP sheet, and Table 1 gives the material properties [8]. To produce a composite sample, the layup was placed between two pieces of PTFE coated glass fabric paper, and gradually wrapped and tightened on two flat steel plates $(320 \times 50 \times 3 \mathrm{~mm})$ using heat-shrink tape. Foldback clips were then used to lock the layup before curing in a pre-heated fan-assisted oven at $225{ }^{\circ} \mathrm{C}$ for 5 hours. After the mould was released, each sample was manually cut into dog-bone shape in readiness for testing. The composite has a fibre volume fraction, $V_{\mathrm{f}}$, of $30 \%$.

Table 1 Material properties of glass fabric and polypropylene sheet.

\begin{tabular}{lcc}
\hline Materials & GF plain-weave woven & PP sheet \\
\hline Density & $200 \mathrm{~g} / \mathrm{m}^{2}$ & $0.9 \mathrm{~g} / \mathrm{cm}^{3}$ \\
Thickness $(\mathrm{mm})$ & 0.20 & 0.5 \\
Construction warp $\times$ weft $(\mathrm{th} / \mathrm{cm})$ & $7.4 \times 7.4$ & -- \\
Poisson's ratio & 0.2 & 0.42 \\
Elastic modulus $(\mathrm{GPa})$ & 72.4 & 1.53 \\
Shear modulus $(\mathrm{GPa})$ & 30 & 0.54 \\
Thermal expansion coefficient & 5.0 & 84.8 \\
$\left(\mathrm{TEC} \mu \mathrm{m} / \mathrm{m}^{\circ} \mathrm{C}\right)$ & & \\
\hline
\end{tabular}

\section{B. Macroscopic failure characterisation}

Macroscopic shear failure of the GFPP composite was performed at the ENGIN-X of Rutherford Appleton Laboratory, UK, following ASTM D3518 [25]. Figure 3 shows the experimental setup. Composite samples $\left( \pm 45^{\circ}\right.$ of layup) were clamped to the tensile grips inside an environmental chamber through bespoke aluminium adapters with a span of $30 \mathrm{~mm}$. An extensometer and a $k$-type thermocouple were mounted on the sample surface. All tests were performed at a crosshead speed of $2 \mathrm{~mm} / \mathrm{min}$ at ambient temperature. Owning to limitation on the space of environmental chamber, the applied strain level was limited to $30 \%$.

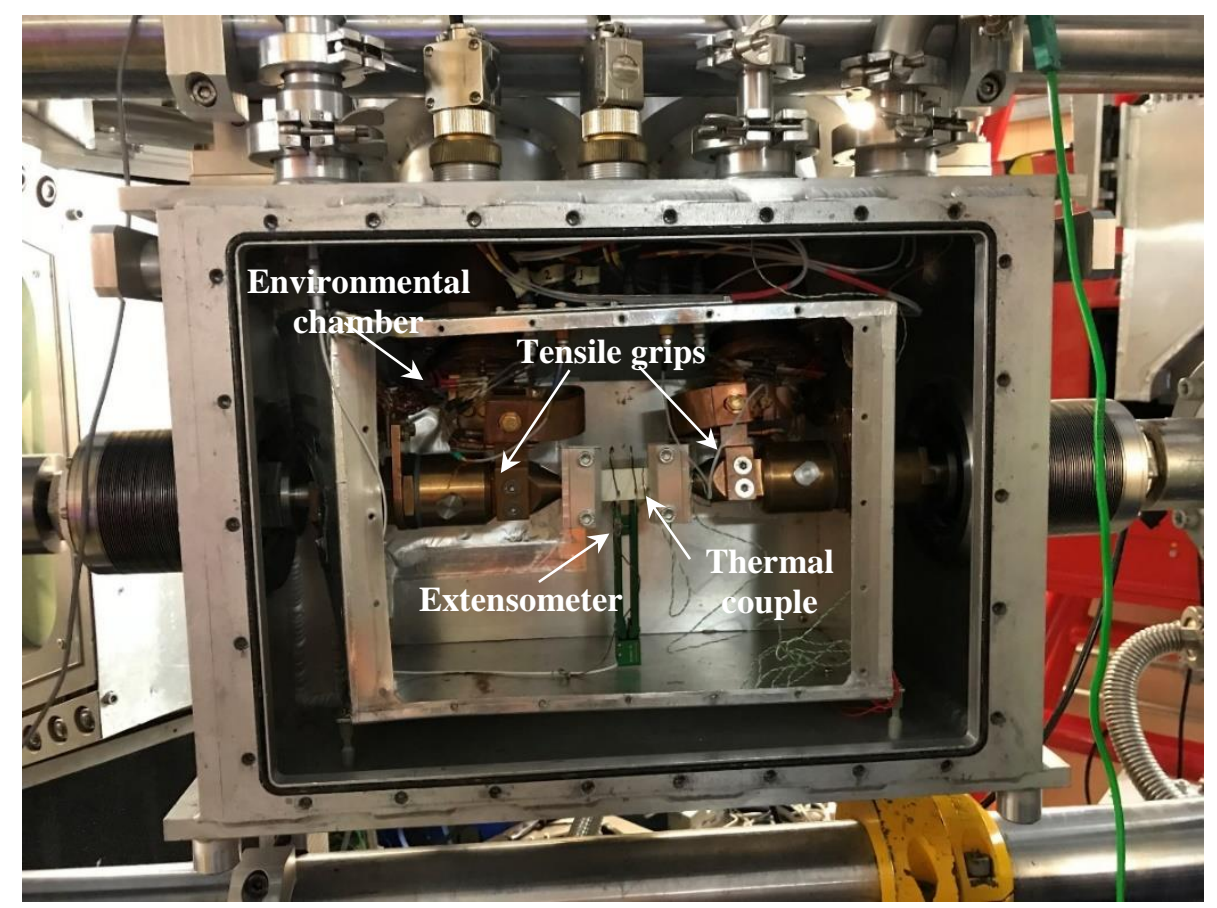

Figure 3 Experimental setup for macroscopic shear failure tests at EINGIN-X. 
The compliance in the fibre direction is virtually independent of time effects; the compliance components $S_{12}$ and $S_{21}$ have weak time dependencies [26]. The fibre-dominated compliance terms $\left(S_{11}=S_{22}, S_{12}=S_{21}\right)$ are assumed to be time independent and the matrix dominated term $\left(S_{66}\right)$ is time dependent. For an orthotropic woven composite under plane stress, the stress-strain relationship is:

$$
\left[\begin{array}{c}
\varepsilon_{11}(t) \\
\varepsilon_{22}(t) \\
\gamma_{12}(t)
\end{array}\right]=\left[\begin{array}{ccc}
S_{11} & S_{12} & 0 \\
S_{12} & S_{22} & 0 \\
0 & 0 & S_{66}(t)
\end{array}\right]\left[\begin{array}{c}
\sigma_{1}(t) \\
\sigma_{2}(t) \\
\tau_{12}(t)
\end{array}\right]+\left[\begin{array}{c}
0 \\
0 \\
\gamma_{12}(t)
\end{array}\right]_{v i s}
$$

where $S_{\mathrm{ij}}$ are the elements of the compliance matrix; the subscript ${ }_{\text {vis }}$ is the viscoelastic term of strain. In-plane shear properties of a composite can be determined through a uniaxial tensile test from $\pm 45^{\circ}$ laminates following ASTM D3518 [25]. Thus, the viscoelastic strain follows:

$$
\gamma_{12}(t)_{v i s}=\gamma_{12}(t)-\tau_{12}(t) / G_{12}
$$

\section{Microscopic failure characterisation}

The NSS was also performed using the ENGIN-X as shown schematically in Figure 4. A bespoke tensile adapter was used to clamp the composite samples, with a span of $30 \mathrm{~mm}$ to allow the scattered neutrons passing through. The incident neutron beam was $45^{\circ}$ to the longitudinal axis of the sample, and the gauge volume of the beam was $4 \times 4 \times 10 \mathrm{~mm}$. Strain was applied through an Instron machine, at a constant displacing speed of $2 \mathrm{~mm} / \mathrm{min}$. Stress free pure PP (before and after heat treatment), pure GF and GF/PP composite samples, were first scanned as the references for the subsequent stressed samples.

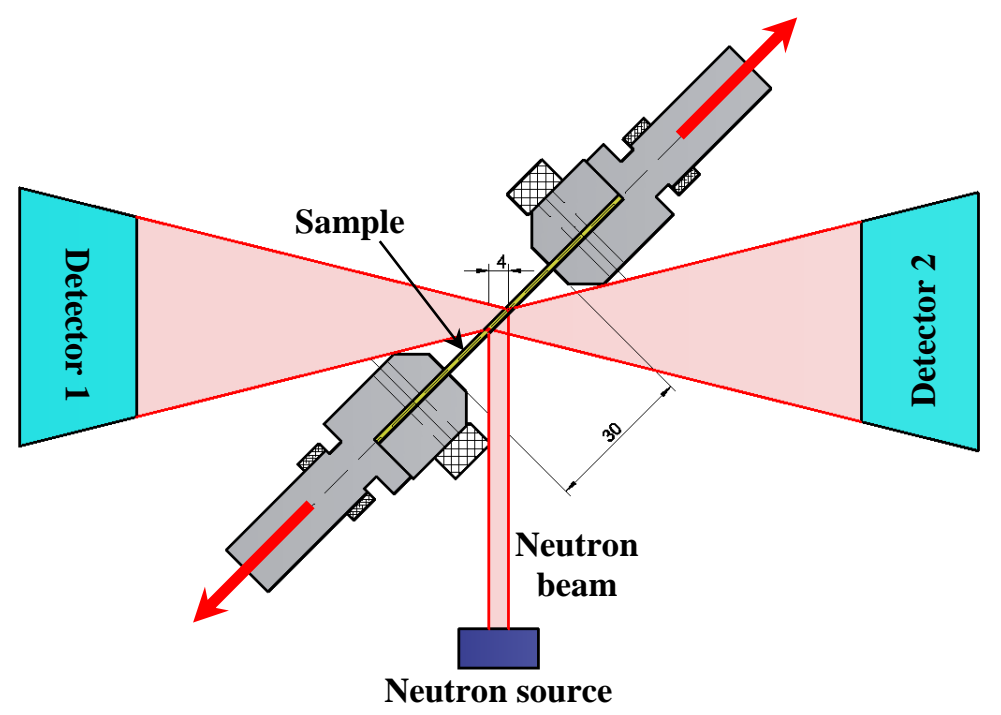

Figure 4 Schematic representation of experimental setup at ENGIN-X, with a sample span of $30 \mathrm{~mm}$.

\section{Results and discussion}

\section{A. Residual stress within a composite}

Since the reinforcement GF has marginal effect on the neutron pattern, residual stress within a composite can be evaluated through microstructural changes within the PP matrix. The oven-baking process corresponds to recrystallisation of PP, the heat treated PP only sample has a crystallinity of $73.3 \%$, and $\beta$-crystal ratio of $53.4 \%$. When solidified into a composite, there is slight reduction in both crystallinity and $\beta$-crystal ratio, giving $70.9 \%$ and $51.0 \%$, respectively. Comparison of neutron patterns from both samples show slightly peak shifts of (111) and (1)31) crystalline planes, which belong to diffractions of $\alpha$-crystals. Both interplanar distances become bigger, indicating the residual stresses introduce tension in the composite as $\alpha$-crystals are under compression. Residual strain associated with these two peaks are 3\% and 5\%, respectively. These residual stresses are caused by mismatch of thermal expansion coefficients between fibre and matrix materials during cooling [27]. 


\section{B. Macroscopic shear failure}

Figure 5 shows a shear stress-strain curve of a GF/PP composite at room temperature. The shear modulus, $G_{12}$, is calculated from the quasi-elastic linear region, and gives $851 \mathrm{MPa}$. It then becomes nonlinear, followed by a second almost linear region, giving a lateral shear modulus $G_{12}^{*}=87.6 \mathrm{MPa}$, before failure occurs. The shear strength and shear strain at macroscopic failure are $48.0 \mathrm{MPa}$ and $27.3 \%$, respectively.

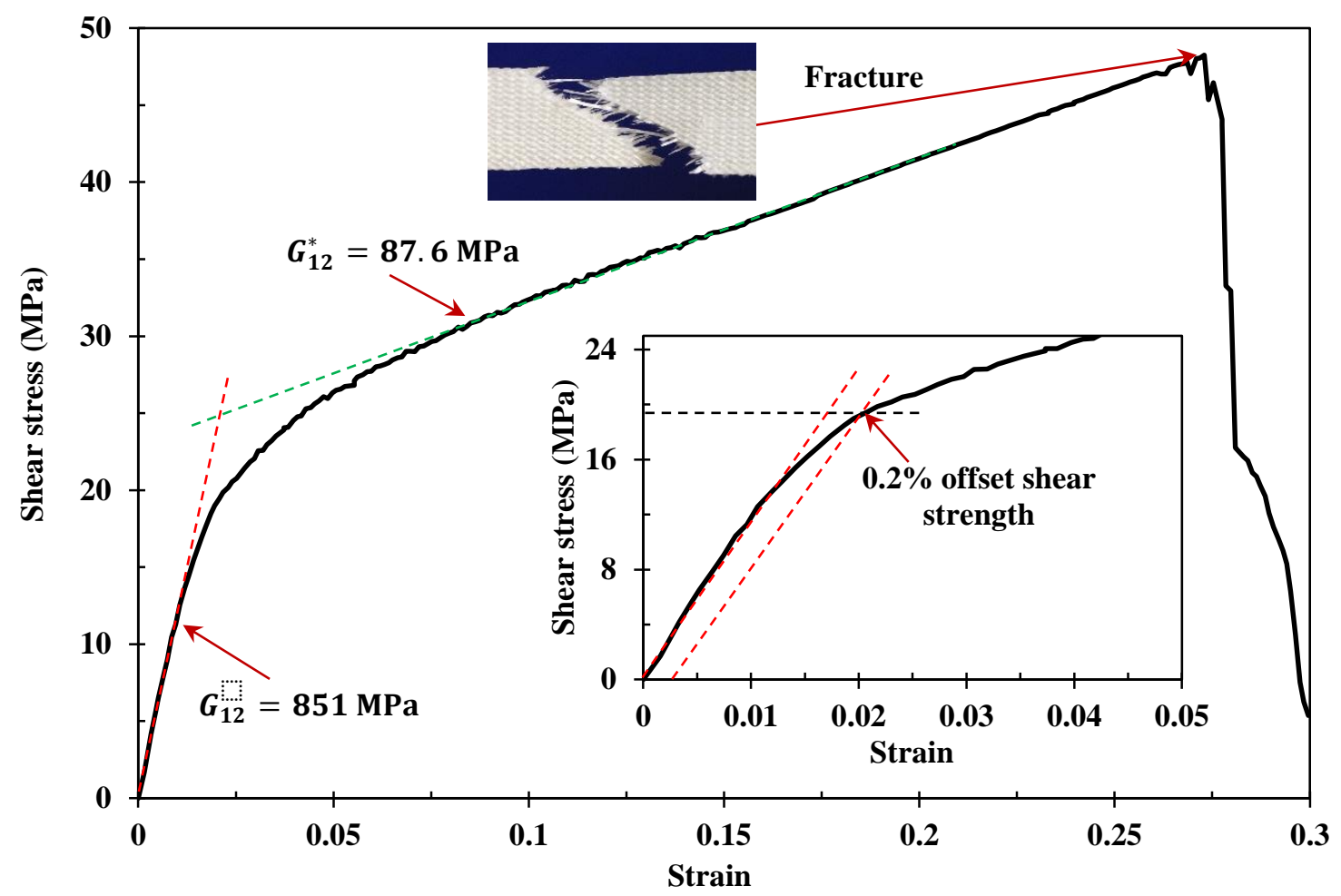

Figure 5 Shear stress-strain curve of a GF/PP sample tested at room temperature: inset shows the definition of $0.2 \%$ offset shear strength following ASTM D3518 [25].

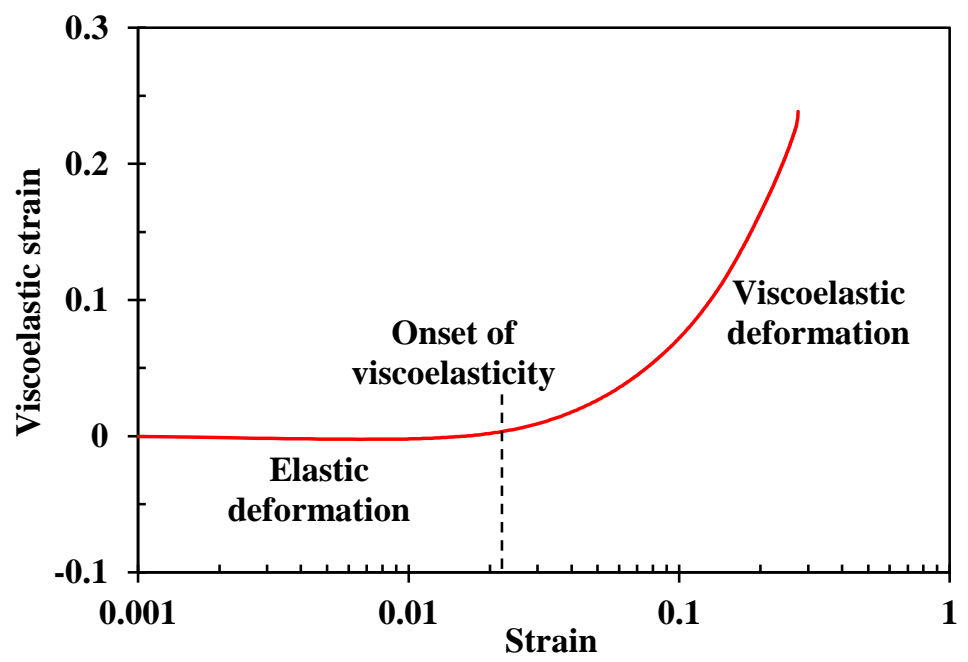

Figure 6 Plot of viscoelastic strain change during shear failure of a GF/PP composite sample at room temperature. 
The yield shear stress and strain can be determined through offsetting the quasi-elasticity line by $0.2 \%$ along the strain axis as recommended by ASTM D3518 [25]. Thus, the transition from elastic to viscoelastic deformation is at a strain level of $2.2 \%$, with a yield stress of $19.0 \mathrm{MPa}$. The growth of viscoelastic strain can be determined following Eq. 8, resulting data show in Figure 6. The elastic region corresponds to the quasi-elastic linear region as in Figure 5. The secondary linear region (as in Figure 5) correlates to the significant increase in viscoelastic strain as shown in Figure 6: this is consistent with the linear viscoelasticity of GF/PP composite as observed from the stress relaxation tests (experimental data are deposited in [28]).

\section{Microscopic shear failure}

Figure 7 shows a comparison of neutron patterns of GF/PP composite at three different strain levels at room temperature. Generally there are peak shifts of (111) and (131) crystalline planes from $\alpha$-crystals when stressed. Figure 8 shows more detailed crystalline peak evolutions from both $\alpha$ - and $\beta$-crystals. The (representative) diffraction peak (300) of $\beta$-crystals is stable across all of the applied strain levels, where $\beta$-crystals are primarily un-oriented [24]. The $\alpha$-crystals endure tensile strains in response to tensile loading, which is observed under all testing temperatures [28]. Thus, the microstructural analysis is mainly focused on phase evolution of $\alpha$-crystals.

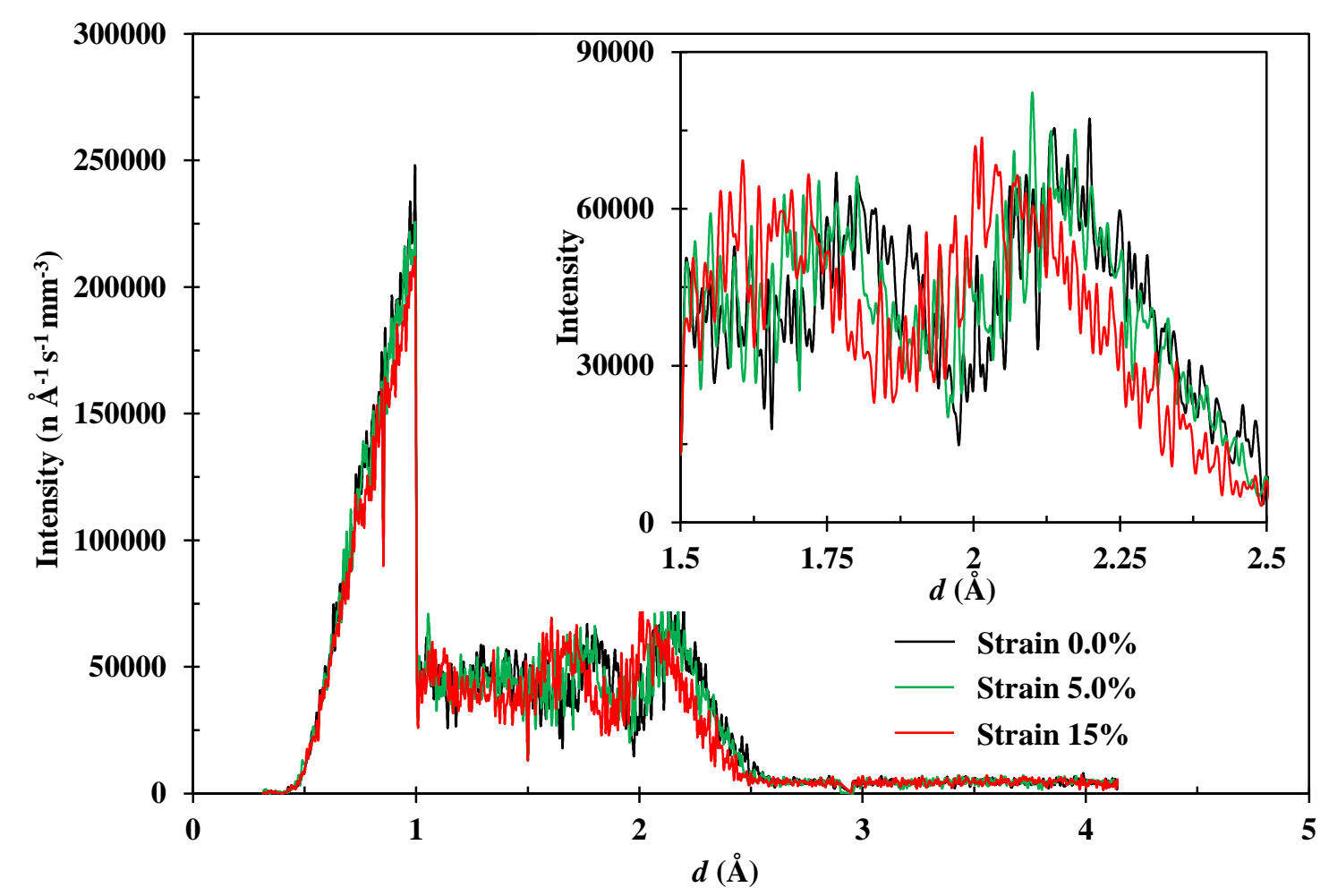

Figure 7 Comparison of neutron patterns of GF/PP composite at different strain levels under room temperature.

Figure 8 shows more details of the changes in peak positions of both (111) and (131). They both reduce close to linear with applied macroscopic strain, and contribute to the elastic responses of the composite; the reductions then start to slow down at $\sim 20 \%$ of applied strain. Further deformation corresponds to saturated and stabilised peak positions of $\alpha$-crystals, where extra deformation relies mainly on deforming in the amorphous or oriented amorphous regions as they are relatively softer than crystals. 

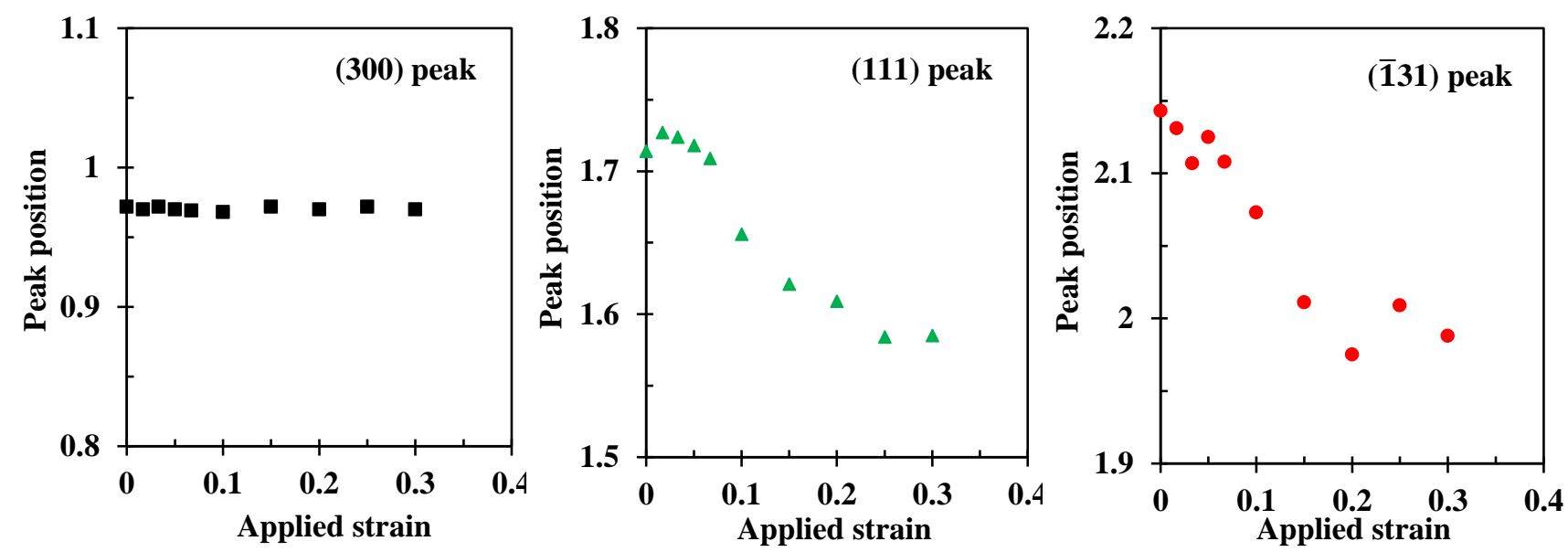

Figure 8 Crystalline peak position changes with applied strain: (111) and (131) peaks are from $\alpha$-crystals; the (300) peak is representative of $\beta$-crystals.

\section{Failure mechanisms}

The crystalline peak positions as discussed above are monitored in longitudinal direction $\left(q_{1}\right.$ as in Figure 1), elastic strain carried by $\alpha$-crystals can be calculated using Eq. 3. Figure 9 shows the microstructural changes in terms of strain, crystallinity and ratio of $\beta$-crystals. The scatter of data points may be attributed to discontinuous macroscopic strain steps and to material variation. The premature failure of crystals was determined from the neutron pattern, where peak spallation was clearly observed at a certain strain value. When crystal deformation initiates, the strain carried by crystals increases almost linearly with applied macroscopic strain; fracture is observed at macroscopic strain of $15 \%$ : this is in accordance with significant drop in crystallinity as in Figure 9-b; the strain carried by the crystals then becomes saturated and stabilised at around 14\%. Upon initial loading, it is also observed that there is an increase in $\beta$-crystal ratio, since applying shear can promote the growth of $\beta$-crystals [29], which then decreases proportionally with macroscopic strain.
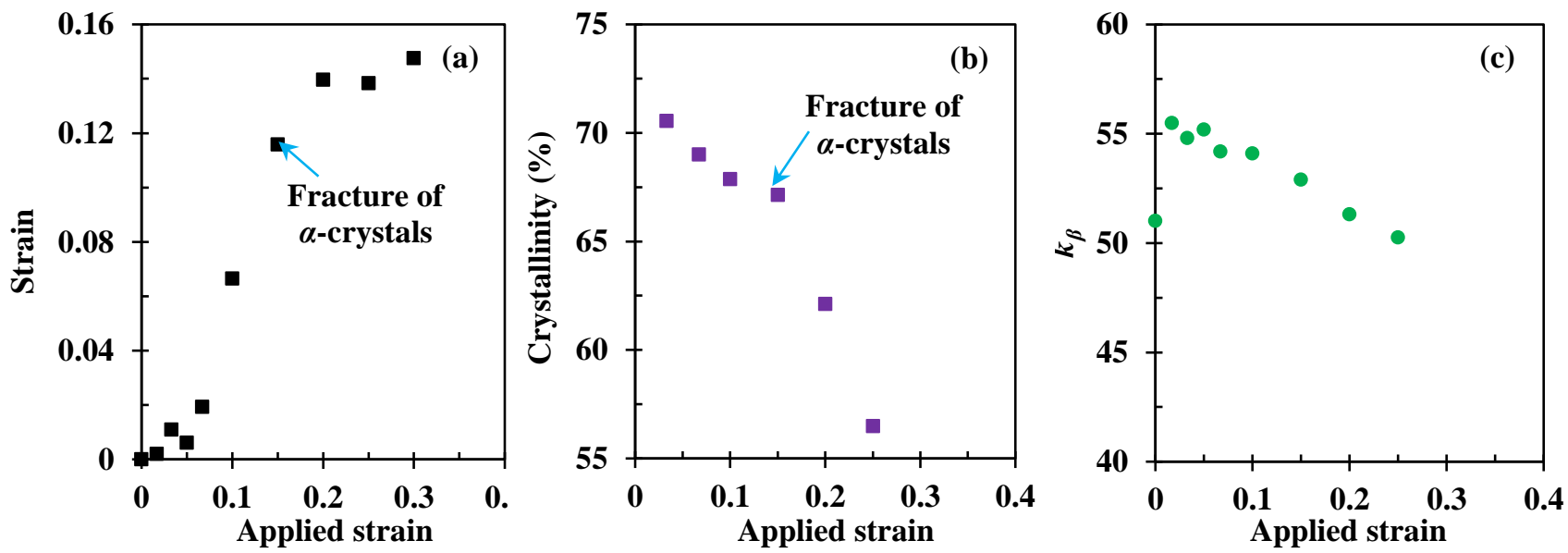

Figure 9 Microstructural changes of GF/PP composite at room temperature, showing (a) longitudinal elastic strain carried by $\alpha$-crystals, (b) crystallinity and (c) ratio of $\beta$-crystals, $k_{\beta}$; fracture is determined from clear observation of peak spallation in the neutron pattern.

To further understand the micromechanical evolution during loading, a microstructural model of polymeric matrix is essential. For semi-crystalline material, a two-phase microfibrillar model was developed by Peterlin [30], and is generally considered to be applicable. Here, it is considered that crystalline and amorphous phases are bridged by taut-tie molecular chains (TTMs), which have a distribution of contour lengths or strains at breaking, and carry loads when stressed [31]. Later, the TTMs were regarded as in the form of oriented amorphous phase, giving the three- 
phase or 'Swiss-cheese' model [32], and proved to control the instantaneous mechanical performance, such as stiffness and toughness in some materials [33, 34].

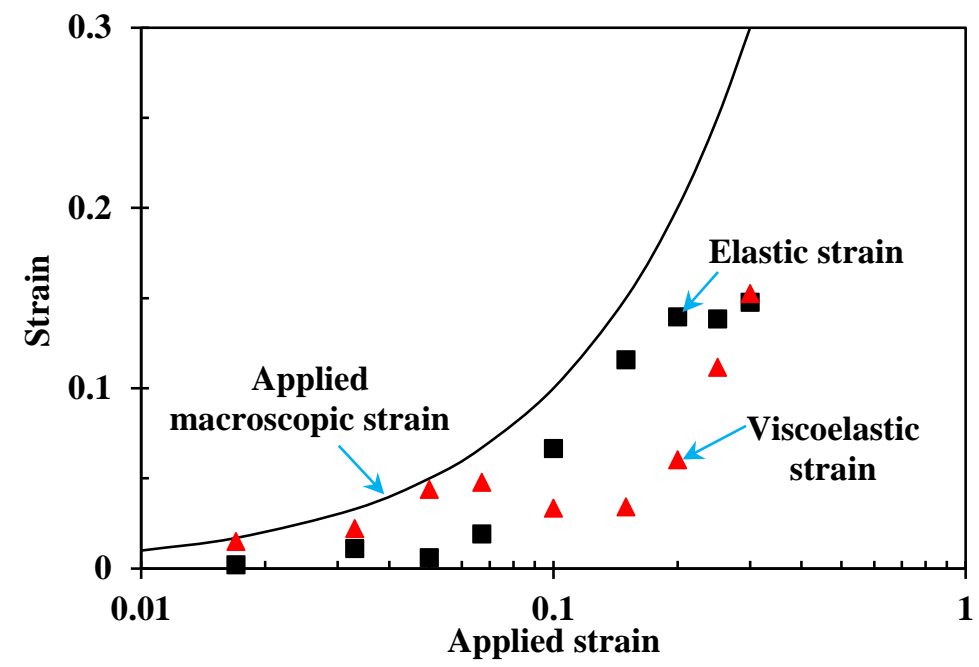

Figure 10 Microstructural strain evolution in response to applied macroscopic strain of GF/PP composite at room temperature, showing changes of both elastic and viscoelastic strains.

Figure 10 shows the evolution of elastic and viscoelastic strain at micromechanical level, together with the applied macroscopic strain. Since elastic strain is directly from crystalline peaks, viscoelastic strain represents deformations from both amorphous and oriented amorphous regions. It infers that at low macroscopic strain, i.e. from 0 to $5 \%$, the crystals are reluctant to the mechanical loading: only $0.6 \%$ out of $5 \%$ of applied strain is carried by $\alpha$-crystals. This indicates that initial loads are carried by TTMs. The yield strain of GF/PP composite (at macroscopic level) is determined to be $2.2 \%$ (Section V.B.), which attributes to the maximum capacity of the oriented amorphous phase. This is in consistence with previous findings as the TTMs determine instant load-carrying capacity in some material systems [31]. At intermediate strain level, from 5\% to 15\%, load transitions occur within all three phases. Elastic deformations are mainly carried by crystals and proportional to the applied macroscopic strain (Figure 10); viscoelastic (or residual) strains are stored mainly in the amorphous phase which control the viscoelasticity [34]. Further loading leads to fracture of crystals, signifying that the maximum capacity of $\alpha$-crystals carried by straining becomes stabilised; there is then a significant drop in crystallinity, as additional deformation relies mainly on amorphous regions.

\section{Conclusions}

We have investigated in-situ, the micromechanical shear failure of a GF/PP composite using ENGIN-X neutron diffractometer at Rutherford Appleton Laboratory. The shear failures at both macroscopic and microscopic levels are determined to provide further insight into the failure mechanisms of the composite.

The micromechanical failure of the GF/PP composite is dominated by the PP matrix, which follow the three-phase microstructural model as consisting of crystalline, amorphous and oriented amorphous phases. There are clear observations of microstructural phase changes when subjected to loading. It is found that, at room temperature, the oriented amorphous phases are the dominant load bearing constituent within the composite. Since macroscopic yielding occurs before the appearance of any fracture of microstructures, it is concluded that the yield is controlled by the TTMs, and therefore the load-carrying capacity of the GF/PP composite. As an established material system for CTS structures, these new findings provide valuable information for optimisation of manufacturing process, and maintain structural integrity of the CTS on folding.

Microstructural phase transitions within a semi-crystalline material are usually reversible, and dependent on external conditions. Future work will focus on temperature dependency, stress relaxation, as well as endurance analysis, in order to facilitate structural design and service life predictions of the CTS in aerospace applications. 


\section{Acknowledgements}

We acknowledge financial support from Innovate UK (Grant No. 113077, RG82506), in partnership with SAFRAN Landing Systems Ltd., UK. The award of ENGIN-X beamtime (RB1910213) from the ISIS Neutron and Muon Source, Rutherford Appleton Laboratory, Science and Technology Facilities Council, UK, is also acknowledged. We also thank the technical support from the Materials and Structures Laboratories, Cambridge University Engineering Department.

\section{References}

[1] Daton-Lovett A. An extendible member. PCT/GB97/00839, 1996.

[2] Iqbal K, Pellegrino S. Bi-stable composite shells. Proceeding 41st AIAA/ASME/ASCE/AHS/ASC Struct. Struct. Dyn. Mater. Conf. Exhib., Atlanta: 2000, p. 1-8.

[3] Galletly DA, Guest SD. Bistable composite slit tubes. I. A beam model. Int J Solids Struct 2004; 41: 451733.

[4] Yee JCH, Pellegrino S. Folding of woven composite structures. Compos Part A Appl Sci Manuf 2005; 36: 273-8.

[5] Guest SD, Pellegrino S. Analytical models for bistable cylindrical shells. Proc R Soc A Math Phys Eng Sci 2006; 462: 839-54.

[6] Schmidt RK. Aircraft landing gear assembly. EP3069991B1, 2017.

[7] Seffen KA, Wang B, Guest SD. Folded orthotropic tape-springs. J Mech Phys Solids 2019; 123: 138-48.

[8] Wang B, Seffen KA, Guest SD. Folding of bistable composite tape-springs. University of Cambridge, Department of Engineering; 2019.

[9] Wang B, Seffen KA, Guest SD. Shape of a bistable composite tape-spring in folding. AIAA SciTech 2019 Forum, San Diego: 2019, p. 1-12.

[10] Fitzpatrick ME, Lodini A. Analysis of residual stress by diffraction using neutron and synchrotron radiation. CRC Press; 2003.

[11] Hutchings MT, Withers PJ, Holden TM, Lorentzen T. Introduction to the characterization of residual stress by neutron diffraction. CRC press; 2005.

[12] Wang B, Zhong S, Lee T, Fancey KS, Mi J. Non-destructive testing and evaluation of composite materials/structures: A state-of-the-art review. Adv Mech Eng 2020: under review.

[13] Santisteban JR, Daymond MR, James JA, Edwards L. ENGIN-X: a third-generation neutron strain scanner. J Appl Crystallogr 2006; 39: 812-25.

[14] Chadwick J. Possible existence of a neutron. Nature 1932;129:312. doi:10.1038/129312a0.

[15] Natta G, Corradini P. Structure and properties of isotactic polypropylene. Nuovo Cim 1960; 15: 40-51.

[16] Stamm M, Schelten J, Ballard DGH. Determination of the chain conformation of polypropylene in the crystalline state by neutron scattering. Colloid Polym Sci 1981; 259: 286-92.

[17] Meille SV, Brückner S. Non-parallel chains in crystalline $\gamma$-isotactic polypropylene. Nature 1989; 340: 455.

[18] Turner-Jones A, Aizlewood JM, Beckett DR. Crystalline forms of isotactic polypropylene. Die Makromol Chemie Macromol Chem Phys 1964; 75: 134-58.

[19] Mollova A, Androsch R, Mileva D, Gahleitner M, Funari SS. Crystallization of isotactic polypropylene containing beta-phase nucleating agent at rapid cooling. Eur Polym J 2013; 49: 1057-65.

[20] Turner-Jones A. Development of the $\gamma$-crystal form in random copolymers of propylene and their analysis by dsc and X-ray methods. Polymer (Guildf) 1971; 12: 487-508.

[21] Hall MM, Veeraraghavan VG, Rubin H, Winchell PG. The approximation of symmetric X-ray peaks by Pearson type VII distributions. J Appl Crystallogr 1977; 10: 66-8.

[22] Immirzi A. Constrained powder-profile refinement based on generalized coordinates. Application to X-ray data of isotactic polypropylene. Acta Crystallogr Sect B 1980; 36: 2378-85.

[23] Varga J, Karger-Kocsis J. Interfacial morphologies in carbon fibre-reinforced polypropylene microcomposites. Polymer (Guildf) 1995; 36: 4877-81.

[24] Somani RH, Hsiao BS, Nogales A, Fruitwala H, Srinivas S, Tsou AH. Structure development during shear flow induced crystallization of i-PP: In situ wide-angle X-ray diffraction study. Macromolecules 2001; 34: 5902-9.

[25] ASTM International. ASTM D3518 - Standard test method for in-plane shear response of polymer matrix composite materials by tensile test of a $\pm 45^{\circ}$ laminate 2013:1-7.

[26] Sullivan JL. Creep and physical aging of composites. Compos Sci Technol 1990; 39: 207-32.

[27] Hull D, Clyne TW. An introduction to composite materials. Cambridge university press; 1996. 
[28] Wang B, Seffen KA, Guest SD, Lee TL, Huang S, Luo S, et al. In-situ studies of failure mechanisms of a bistable composite tape-spring under extreme conditions. STFC ISIS Neutron Muon Source Data J 2019.

[29] Huang M-R, Li X-G, Fang B-R. $\beta$-nucleators and $\beta$-crystalline form of isotactic polypropylene. J Appl Polym Sci 1995;56:1323-37.

[30] Peterlin A. Molecular model of drawing polyethylene and polypropylene. J Mater Sci 1971; 6: 490-508.

[31] Peterlin A. Structural model of mechanical properties and failure of crystalline polymer solids with fibrous structure. Int J Fract 1975; 11: 761-80.

[32] Prevorsek DC, Harget PJ, Sharma RK, Reimschuessel AC. Nylon 6 fibers: Changes in structure between moderate and high draw ratios. J Macromol Sci Part B 1973; 8: 127-56.

[33] Marcellan A, Bunsell AR, Laiarinandrasana L, Piques R. A multi-scale analysis of the microstructure and the tensile mechanical behaviour of polyamide 66 fibre. Polymer (Guildf) 2006; 47: 367-78.

[34] Wang B, Fancey KS. Viscoelastically prestressed polymeric matrix composites: An investigation into fibre deformation and prestress mechanisms. Compos Part A Appl Sci Manuf 2018; 111: 106-14. 